\title{
Review of Morphology Dependent Charge Carrier Mobility in MEH-PPV
}

\author{
A. R. Inigo, ${ }^{\mathrm{a}, \mathrm{b} *}$ Y. F. Huang ( 黃一芳), J. D. White ${ }^{\mathrm{a}, \mathrm{c} *}$ ( 白小明),

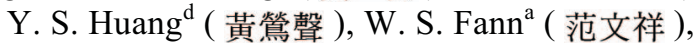 \\ K. Y. Peng ( 彭剛勇) and S. A. Chen ${ }^{\mathrm{e}}$ ( 陳壽安) \\ ${ }^{\mathrm{a}}$ Institute of Atomic and Molecular Sciences, Academia Sinica, and Department of Physics, \\ National Taiwan University, Taipei 106, Taiwan, R.O.C. \\ ${ }^{\mathrm{b}}$ Nanoelectronics Center, Advanced Technology Institute, University of Surrey, Guildford, Surrey, GU2 7XH, U.K. \\ ${ }^{\mathrm{c}}$ Department of Photonics Engineering, Yuan Ze University, Chung-Li 320, Taiwan, R.O.C. \\ ${ }^{\mathrm{d}}$ Department of Electronic Engineering, National Taiwan University of Science and Technology, \\ Taipei 106, Taiwan, R.O.C. \\ ${ }^{e}$ Department of Chemical Engineering, National Tsing-Hwa University, Hsinchu 300, Taiwan, R.O.C.
}

\begin{abstract}
Charge carrier mobility in poly(2-methoxy,5(2'-ethyl-hexyloxy)-p-phenylene vinylene) (MEH-PPV) films were measured as a function of temperature and electric field parallel and perpendicular to the substrate for devices prepared from different solvents and under different processing conditions. Bulk structural morphology was characterized by various $\mathrm{X}$-ray diffraction measurements such as wide angle, small angle and X-ray reflection. Surface morphology was characterized using various scanning probe microscopic techniques. Mobilities were found to follow Gaussian disorder model (GDM) and to be highly anisotropic not only depending on the solvents used but also on the film preparation method such as spin-coating or drop-casting. While no direct correlation was found between charge carrier mobility and photoluminescence, charge transport parameters were correlated with structural morphology.
\end{abstract}

Keywords: MEH-PPV; TOF; TrEL; FET; Morphology; Drift mobility.

\section{INTRODUCTION}

Excellent device characteristics, easy processing conditions, the promise of potential application in low cost lighting and solar energy conversion are a few of the driving factors of science and technological achievements in organic semiconducting polymer materials. ${ }^{1-4} \mathrm{MEH}-\mathrm{PPV}$ is one these materials which is widely used as a light emitting material for displays. Extensive studies have focussed on the correlation between photophysical properties and morphology. ${ }^{5-8}$ It has been well established that the morphology of the polymer influences the photophysical properties and hence the device characteristics. These morphology studies investigated as a stand alone were used to explain the device characteristics that related charge transport and morphology. ${ }^{9-12}$ We have extended these studies to working device configurations such as diodes and transistors made from MEH-PPV.

Charge carrier mobility can be measured by various methods such as time of flight (TOF), ${ }^{13-17}$ transient electroluminescence (TrEL), ${ }^{18-19}$ and field effect transistors
(FET) ${ }^{20-21}$ While each of these three methods gives different mobility values due to its different sample and measurement configuration requirements, combined analysis with structural parameters provides valuable information about the material system being investigated. i.e., the TOF method provides charge transport information in the plane perpendicular to the substrate for thick samples $(\sim \mu \mathrm{m}$ thickness). ${ }^{15}$ TrEL also provides information about the mobility in the plane perpendicular to the substrate, but for thin samples ( $\sim 100 \mathrm{~nm}$ thick). FET provides information about mobility parallel to the substrate for both thick and thin films. (For the latter it is preferable to use a bottom electrode configuration as bottom gate as top source and drain electrodes may not work with thick films due to the low mobility nature of polymers). By making use of these three methods to measure mobility, all the necessary information to understand the physics of charge transport in MEH-PPV can be obtained.

While the charge transport measurements provide first hand information about the charge transport sites' spa-

Dedicated to the memory of Professor Wunshain Fann (1961-2008). 
tial and energetic distribution, bulk and surface morphology studies are required to arrive at a comprehensive relevant structural model which can explain both morphological and charge transport investigations. Wide angle X-ray diffraction (WAXS) has been widely used to obtain information about the nanometer domain structures. ${ }^{22}$ As these polymers tend to form a mixture of amorphous and crystalline structures, small angle X-ray diffraction (SAXS) is needed to provide information about mesoscale structures. $^{23}$

In this paper, we review the morphology (bulk and surface) dependent charge carrier mobility with respect to solvents (used for device fabrications), temperature (annealing), film fabrication process (spin cast, drop cast), and charge transport direction (parallel and perpendicular to the substrate).

\section{EXPERIMENTAL}

Thin films (of the order of 25-200 nm) were prepared by spin-coating. Thick films (of the order of $\mu \mathrm{m}$ ) were prepared by drop casting. In the following discussion, drop-cast films prepared under the influence of electric field will be denoted as E-field-cast or E-cast for short, while those films which were first drop casted and then annealed will be referred to as annealed drop cast films.

In all cases, indium tin oxide (ITO) coated glass substrates were first cleaned by treatment with organic solvents in an ultrasonic bath and finally with acetone and oxygen plasma. MEH-PPV was dissolved in either chlorobenzene or toluene and drop-cast onto the prepared substrates in a solvent rich atmosphere to get high optical quality films. In the case of E-cast films, an electric field was applied during the fabrication process as outlined in detail in Ref. 24. The coated films were dried for 12 hours at room temperature and the residual solvent was removed by dynamic vacuum at $10^{-3} \mathrm{~Pa}$. In the case of annealing, samples were heated in a nitrogen atmosphere at $\sim 475 \mathrm{~K}$ for 12 hours and cooled down slowly to room temperature. Subsequently, samples were placed in a vacuum chamber and a $100 \mathrm{~nm}$ thick Au films were thermally evaporated by the shadow mask procedure to yield an active area of $4 \mathrm{~mm}^{2}$ Thickness of the polymer sample was measured by the Dektak 3030 Surface Profile Measuring System. Capacitances of the devices were measured by a Hewlett Packard 419A Impedance/Gain Phase analyser. Defect densities were determined by the Nuclear Magnetic resonance (NMR) spectroscopy.
For TOF measurements, the $5 \mathrm{~ns}$ pulse at $532 \mathrm{~nm}$ from YAG laser was used as an optical excitation. The photocurrent transients were measured by digital storage oscilloscope (Tektronix TDS 744A). The sample was mounted in vacuum cryostat controlled with temperature, and kept in the vacuum for 12 hours prior to the measurements. At high temperatures, the time resolution of the experimental was limited at short transit times $\left(\mathrm{t}_{\tau}\right)$ by the $\mathrm{RC}$ time constant. At low temperatures and low applied electric fields, the signal to noise ratio was the limiting factor for obtaining data. At high electric fields, dielectric breakdown provided the upper limit. For the results presented here, $\mathrm{RC}<\mathrm{t}_{\tau}$ and the total current injected in to the film was kept around $0.01 \mathrm{CV}$, where $\mathrm{C}$ is the structure capacitance and $\mathrm{V}$ is the applied voltage. The transit time was defined as the intersection of asymptotes to the plateau and the declining slope of the current transient.

Gracing incidence wide angle X-ray scattering (GI-WAXS) and X-ray reflectivity (XRR) measurements were performed at the wiggler beamline BL17B of National Synchrotron Radiation Research Center (NSSRC), Taiwan. XRR data was analysed with various layer models using the Pratt32 software. Two dimensional GI-WAXS patterns were also also recorded on image plates at BL17A for longer wavelengths. Small angle x-ray scattering, SAXS, measurements were performed on the 8-m SAXS instrument at Tsing-Hua University, Taiwan, and also at BL17B, NSRRC (at grazing incidence), with the film surfaces being either perpendicular or parallel to the beam. For SAXS measurements, the sample thin films were detached from the glass substrates and then carefully folded into thick layers of $\sim 120 \mu \mathrm{m}$ thick and $\sim 5 \mathrm{~mm} \times 5 \mathrm{~mm}$ dimensions. X-ray results were corrected for sample transmission, background, detector sensitivity, and normalized to an absolute scattering scale by comparison with a standard sample of polyethylene. The absolute scattering scale calibration makes the intensity comparison between measurements possible. GI-WAXS measurements were also performed at D1 station of Cornell High Energy Synchrotron Source (CHESS), Cornell University, at a wavelength of $1.2 \mathrm{~A}$. Incident angle for gracing incident were kept around $\sim 0.2^{\circ}$ and the scattered photons were detected by charge coupled device (CCD). Data were fitted by Fit2D analysis.

Scanning Electron Microscopy (SEM) images were recorded by use of a field-emission scanning electron microscope (FE-SEM, JEOL JSM-6330TF). Atomic force microscopy images (AFM) were recorded by PISA XE-100 
Table 1. Summary of the Bässler GDM Parameters based on TOF data

\begin{tabular}{lccccc}
\hline Film & $\begin{array}{c}\text { Relative } \mu \\
(0.15 \mathrm{MV} / \mathrm{cm}, 295 \mathrm{~K})\end{array}$ & $\sigma[\mathrm{meV}]$ & $\sum$ & $\mathrm{C}\left[\left(\mathrm{cmV}^{-1}\right)^{-1 / 2}\right]$ & $\begin{array}{c}\mu(0, \infty) \\
{\left[\mathrm{cm}^{2} / \mathrm{Vs}\right]}\end{array}$ \\
\hline Drop-cast from chlorobenzene & 1 & 96 & 2.84 & $4 \times 10^{-4}$ & $2.2 \times 10^{-4}$ \\
Drop-cast from toluene & 5 & 85 & $<1.5$ & $3.3 \times 10^{-4}$ & $1.0 \times 10^{-4}$ \\
E-cast from chlorobenzene & 20 & 73 & $<1.5$ & $6 \times 10^{-4}$ & $0.5 \times 10^{-4}$ \\
Annealed from toluene & 0.15 & & & & \\
Drop-cast from toluene (Defects: 1.8\%) & 6 & 72.6 & 1.48 & & \\
Drop-cast from toluene (Defects: 2.5\%) & 4 & 81.9 & 4.57 & \\
Drop-cast from toluene (Defects: 3.0\%) & dispersive & & & & \\
\hline
\end{tabular}

instrument in non-contact mode using high resonance type cantilevers with Al reflecting coating (Nanosensors). Scanning confocal microscopy (SCM) measurements were made in-house.

\section{BÄSSLER GDM MODEL}

Throughout this paper, the dependence of mobility on electric field and temperature were interpreted by the Guassian disorder model (GDM) proposed by Bässler. ${ }^{16}$ In GDM, a material is characterized by energy disorder $(\sigma)$ and position disorder $(\Sigma)$. The energy disorder describes the distribution of energy levels (DOS) associated with transport and the position disorder describes the fluctuation in the distance and orientation of the hopping sites associated with the hopping carriers. These two parameters are influenced by material morphology which depends not only on chemical structure but also on processing conditions such as temperature treatment and solvent. While the resulting equations do not permit an analytical solution, based on the results of a Monte Carlo simulation, the dependence of mobility on electric field and temperature in the ranges of electric field used in our devices can be approximated by:

$$
\mu(E, T)=\mu_{o} \exp \left(\frac{2 \sigma}{3 \mathrm{k}_{B} T}\right)^{2} \exp \left[\left(\left(\frac{\sigma}{\mathrm{k}_{B} T}\right)^{2}-\Omega^{2}\right) C \sqrt{E}\right](1)
$$

In this equation, $T$ is the absolute temperature $(\mathrm{K}), \mathrm{k}_{\mathrm{B}}$ is the Boltzman constant, $\mu_{o} \equiv \mu(0, \infty)$ is the mobility at zero field and infinite absolute temperature, and $\mathrm{C}$ is an experimental constant that, according to the model, scales with the square root of intermolecular distance. From the model, $\Omega$ is defined as follows: if $\Sigma>1.5, \Omega=\Sigma^{2}$, otherwise $\Omega=$ 2.25. While $\mu_{o}=\mu(0, \infty), \sigma, \Omega$ and $C$, can be obtained by careful fitting of the electric field and temperature dependence of mobility, the approximation $\mathrm{C}=2.9 \times 10^{-4}$ $\left(\mathrm{cmV}^{-1}\right)^{-1 / 2}$, corresponding to an intermolecular distance of
$0.6 \mathrm{~nm}$, is often used.

For data processing, the mobility was measured at various values of applied electric field and temperature. The natural logarithm of the mobility as a function of the square root of the electric field at each temperature was least-squared fit to a line. The temperature dependence of the intercept is given by:

$$
\ln (\mu(0, T))=\ln (\mu(0, \infty))-\left(\frac{2 \sigma}{3 \mathrm{k}_{\mathrm{B}} \mathrm{T}}\right)^{2}
$$

By plotting the intercept as a function of $1 / \mathrm{T}^{2}$, and employing a linear least-squares fit routine both the zero field infinite temperature mobility $\left(\mu_{\mathrm{o}}\right)$ and the energy disorder $(\sigma)$ were obtained.

The temperature dependence of the slope, $\mathrm{S}(\mathrm{T})$, is given by:

$$
\mathrm{S}(\mathrm{T})=\left(\left(\sigma / \mathrm{k}_{\mathrm{B}} T\right)^{2}-\Omega^{2}\right) C
$$

By plotting $\mathrm{S}(\mathrm{T})$ as a function of $\left(\sigma / \mathrm{k}_{\mathrm{B}} \mathrm{T}\right)$ both the value of the constant $\mathrm{C}$ for our system and the position disorder $(\Sigma)$ were obtained.

\section{RESULTS AND DISCUSSION}

\subsection{Influence of Defects}

The effect on charge transport of the density of tetrahedral defects, common defects observed in MEH-PPV, was investigated. As seen in Table 1, non-dispersive transport disappears once the defect densities increase above $\sim 2.5 \%$. In addition, the energy disorder parameters and position disorder calculated with defect densities less than $2.5 \%$ increase with increasing defects concentration. ${ }^{25}$ In other words, at defect densities $>2.5 \%$, charge transport behaviour is dominated by defects. It is only when the defect density is $<2.5 \%$, are the effects of morphology seen in 
the charge transport behaviour. Thus, in the work presented the following sections, high quality, low-defect MEH-PPV was used (Typically $<2 \%$ )

\subsection{Solvent Dependence}

Fig. 1 shows the hole transient of drop-cast MEHPPV devices prepared from the solvents chlorobenzene and toluene. ${ }^{23}$ The transients show non-dispersive behaviours which consist of an initial peak due to RC time constant at the beginning followed by a plateau and than a long declining edge indicating that the hole transport is non-dispersive (ND) for both films. While the transit time of faster arriving carriers can be seen in the linear scale for ND behaviour, the transit times were determined form the $\log i-\log t$ plot.

Fig. 2 shows the temperature dependence of devices prepared from chlorobenzene and toluene from $225 \mathrm{~K}$ to $325 \mathrm{~K}$ at the interval of $10 \mathrm{~K} .{ }^{23}$ The electric field dependence of mobility follows Poole-Frenkel behaviour,

$$
\ln (\mu(\mathrm{E}, \mathrm{T}) / \mu(0, \mathrm{~T}))=\mathrm{SE}^{1 / 2}
$$

where $\mu(\mathrm{E}, \mathrm{T})$ is the mobility of the charge carriers, $\mathrm{E}$ is an
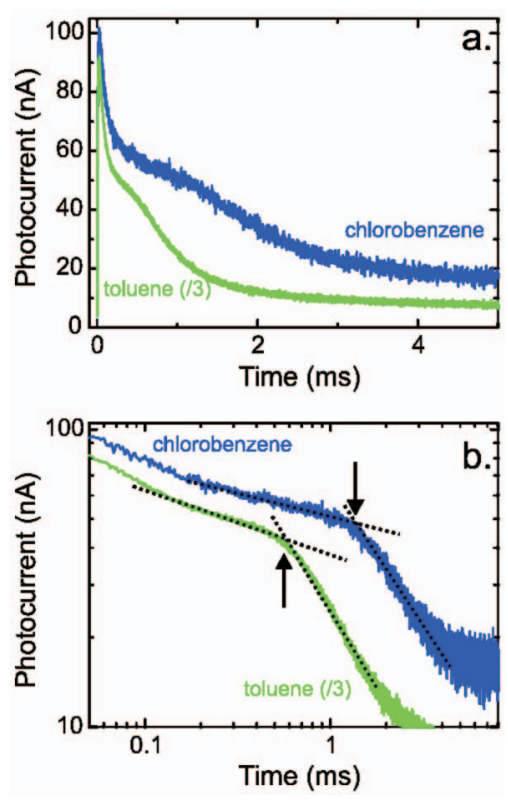

Fig. 1. The photocurrent transients in (a) linear scale and (b) at log-log scale (the arrow points the transit time) at room temperature for films prepared in toluene and in chlorobenzene solutions. The electric field is $1 \times 10^{5} \mathrm{~V} / \mathrm{cm}$. Both films have thickness of $3.7 \mu \mathrm{m}$. externally applied electric field, $\mathrm{S}$ is the slope and $\mu(0, \mathrm{~T})$ is the zero-field mobility. This Poole-Frenkel relationship is observed throughout the range investigated. From the figure, it can be seen that the electric field dependence of mobility is stronger in toluene devices than for chlorobenzene devices at room temperature. In addition, the electric field dependence which is characterised by the slope seems to be varying with the temperatures more in the case of device from chlorobenzene than that of toluene. These are indications that the positional disorder is larger in the device prepared from chlorobenzene than that of toluene. In materials having positional disorder, the charge carriers can diffuse against the direction of applied electric field by detouring around the next favourable charge transporting site. This diffusion assisted detour against the applied electric field results in longer transit time and hence lower mobility. This diffusion process is enhanced at higher temperatures with materials having larger positional disorder. This kind of behaviour is observed in devices prepared from chlorobenzene. The gradual decrease of slope in $\mathrm{S}$ with respect to temperature is characteristic of materials having larger positional disorder. This was quantified by analysis within the GDM model. The GDM energy disorders were calculated as $96 \mathrm{meV}$ and $85 \mathrm{meV}$ for the drop-cast chlorobenzene and toluene by plotting $\ln (\mu(0, T))$ against $1 / \mathrm{T}^{2}$ as shown in Fig.
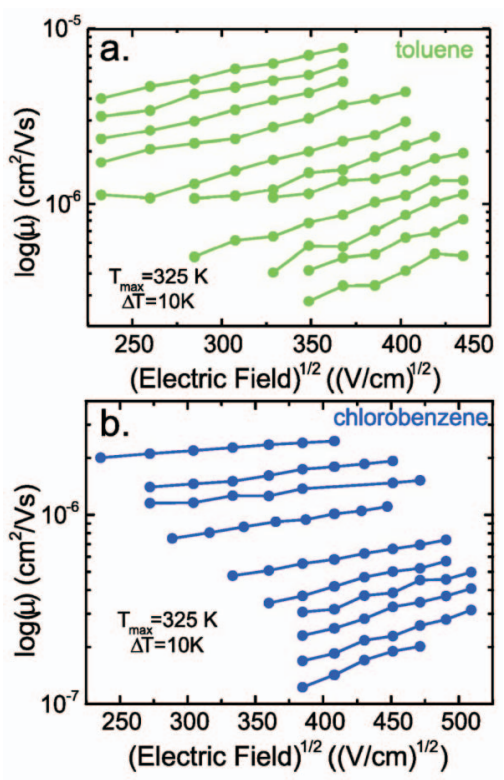

Fig. 2. Temperature and field dependent mobility from $225 \mathrm{~K}$ to $325 \mathrm{~K}$ at the interval of $10 \mathrm{~K}$ for devices drop cast from (a) toluene and (b) chlorobenzene. 
3. The positional disorders were calculated to be 2.84 and $<$ 1.5 for the devices prepared from chlorobenzene and toluene respectively by plotting the slopes of the dependence of mobility as a function of electric field as a function of temperature (Fig. 4).

\subsection{Effect of Thermal Annealing}

The room temperature drop-cast devices exhibited non-dispersive hole transient while the annealed devices exhibited dispersive hole transient. To verify that this is not a thickness dependent effect, film thickness was varied from 1-5 $\mu \mathrm{m}$. The results were similar. This suggests that the dispersive hole transient of annealed devices is not due to a variation in thickness but rather due to a change in morphology affected by the annealing process (which will be discussed later). The electric field dependence of mobility is shown in Fig. $5 .{ }^{14}$ Annealing has reduced the field dependent mobility by approximately one order of magnitude relative to that of drop-cast films.

\subsection{Effect of Applied Electric Field During Deposition}

Fig. 6 compares the field dependent mobility of dropcast and E-field-cast films at room temperature. The solvent in both cases was chlorobenzene. While both follow the Poole-Frenkel relationship as shown in equation (4), ${ }^{24}$ the hole mobilities of E-cast films are one order of magnitude higher than that of drop-cast films. In fact the field dependent mobility at $225 \mathrm{~K}$ in E-field-cast devices are higher than the field dependent mobility of drop-cast devices at $325 \mathrm{~K}$ (Fig. 7). ${ }^{24}$ The value of Poole-Frenkel slope $\mathrm{S}$ is higher in the case of E-field-cast devices than that of drop-cast devices. While this slope gives an indication that the position disorder is higher in drop-cast devices than that

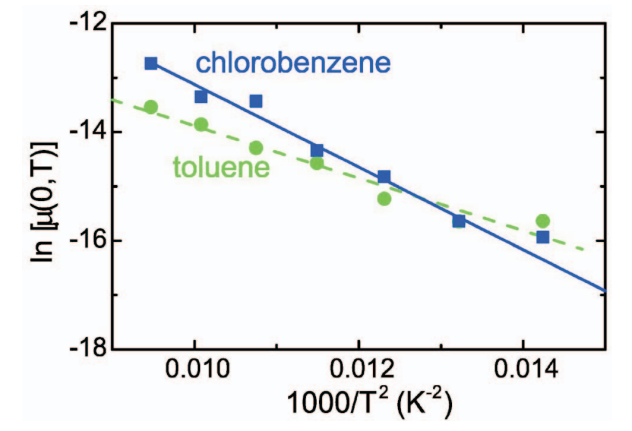

Fig. 3. The zero field mobility is plotted against $1 / \mathrm{T}^{2}$ for devices prepared from (a) toluene and (b) chlorobenzene to determine energy disorder parameter. of E-field-cast, temperature dependent mobility also predicted that the position disorder is indeed higher in drop-cast devices. Quantifying this using GDM, the energy disorder value was calculated as 96 and $73 \mathrm{meV}$ for the drop-cast and E-field-cast devices respectively. The position disorder value were calculated as 2.84 and $<1.5$ for the drop-cast and E-field-cast devices respectively. The field dependent mobility and position disorder values of E-cast

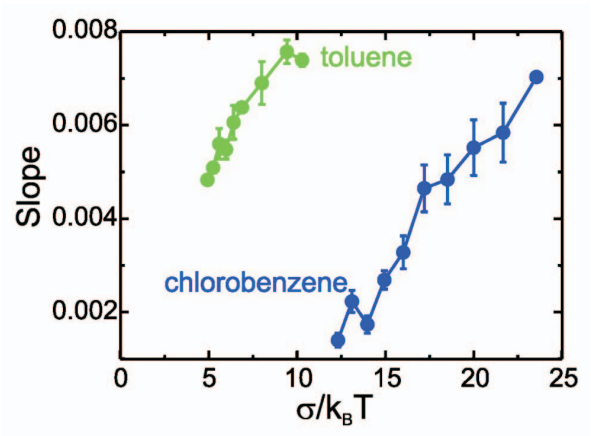

Fig. 4. The slope (S) at different temperatures is plotted against $\left(\sigma / \mathrm{k}_{\mathrm{B}} \mathrm{T}\right)^{2}$ for devices prepared from toluene and chlorobenzene to determine position disorder parameter.

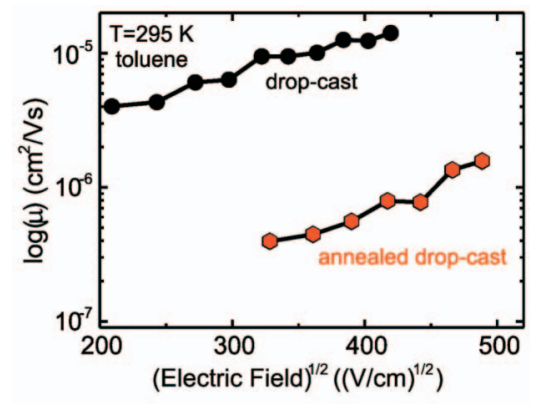

Fig. 5. Room temperature field dependent TOF mobility for drop cast and thermally annealed drop cast devices from toluene.

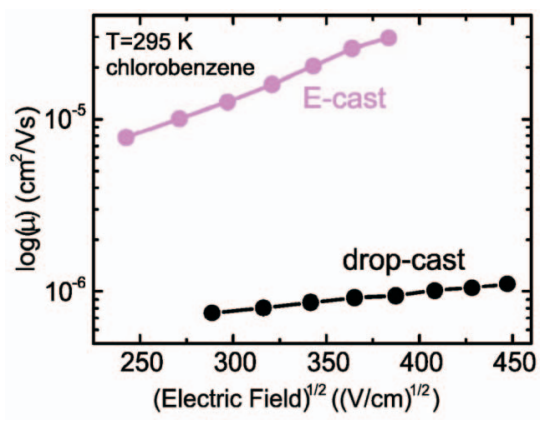

Fig. 6. Temperature dependent field dependent mobilities for the drop cast and E-field cast devices from $225 \mathrm{~K}$ to $325 \mathrm{~K}$ at the interval of $10 \mathrm{~K}$. 
devices prepared from chlorobenzene resembled to the drop-cast devices of toluene from Fig. $2 .^{23}$ These results suggest that position disorder dominates the charge transport characteristics of MEH-PPV and energy disorder effects only become visible in samples having lower position disorders, i.e., for samples having large position disorder, positional disorder dominates charge transport characteristics and affects the change from non-dispersive transport to dispersive transport.

\subsection{Spin-coating vs. Drop-casting}

To evaluate the relevance of mobility values and charge transport characteristics of TOF configuration (thickness in the range 1-5 $\mu \mathrm{m}$ ) and the working OLED configuration (thickness in the range 20-100 nm) we have also measured mobilities by TrEL method. Fig. 8a shows the transient electro-luminescence (TrEL) signal at different applied voltages in logarithmic scale. The transit and delay time decreases as the applied voltage increases. ${ }^{26}$ The field dependent mobilities were calculated from the respective applied voltage dependent transit times. Fig. 8b shows the comparison of room temperature field dependent mobility for the drop-cast, E-cast and spin-cast. ${ }^{26}$ The E-cast devices exhibited mobilities two order of magnitude higher than that spin-cast films and one order of magnitude higher than that of drop-cast films. Clearly spin-processing has changed the morphology of the films relative to the drop cast films.

In order to investigate the morphological origins of this change, the photoluminescence (PL) spectra of the three films were compared. Fig. 8a shows the PL spectra of drop-cast, E-field-cast and spin cast films. ${ }^{26}$ (Note that the PL spectra was the same for films cast from toluene and for chlorobenzene). ${ }^{27}$ The height of the peak around $640 \mathrm{~nm}$

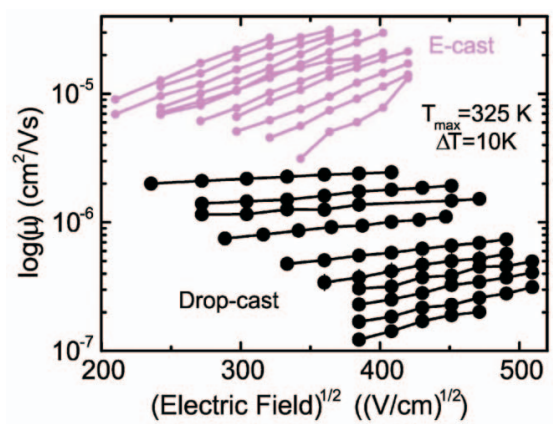

Fig. 7. Temperature dependent field dependent mobilities for the drop cast and E-field cast devices from $225 \mathrm{~K}$ to $325 \mathrm{~K}$ at the interval of $10 \mathrm{~K}$. relative to the main peak indicates the relative amount of interchain interaction present in polymer films, i.e., the higher the peak at $\sim 640 \mathrm{~nm}$ the more interchain interaction and vice versa. In addition to the relative height, the degree of red-shift of $640 \mathrm{~nm}$ peak also reflects the degree of interchain interaction in the film. Both of these indicate that there is less interchain interactions in spin-cast film than in either the drop-cast or E-field cast films.

This mobility difference can be difficult to understand if only the PL, which describes the amount of interchain interaction is considered. Comparing the spin and drop cast films, one might conclude from the PL that the increased mobility in drop cast films relative to spin cast films is due to increased interchain interaction. Conversely, comparing the drop cast and e-cast films, one might from the PL that the increased mobility in e-cast films relative to spin cast films is due to decreased
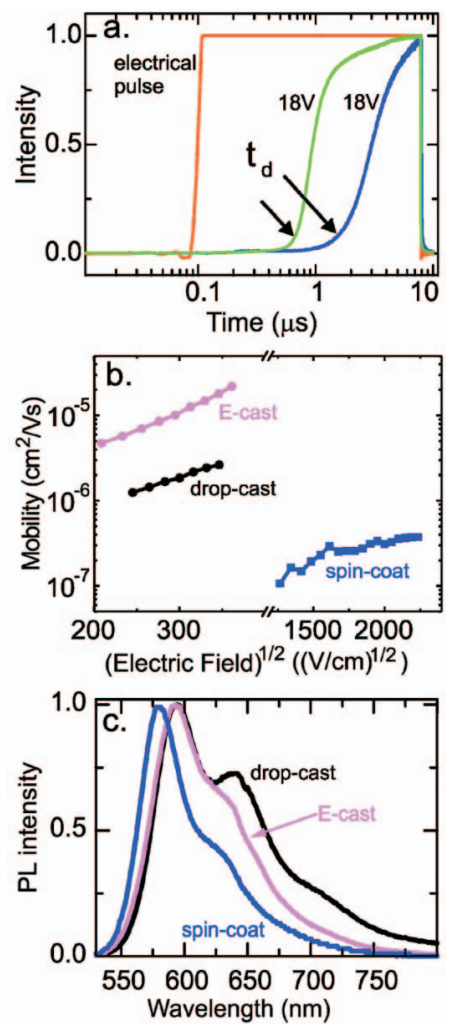

Fig. 8. (a) Transient electroluminescence signal for the spin-cast devices at the applied voltages of $12 \mathrm{~V}(\mathrm{E}=4000 \mathrm{kV} / \mathrm{cm})$ and $18 \mathrm{~V}(\mathrm{E}=6000$ $\mathrm{kV} / \mathrm{cm}$ ) are shown in log-linear plot. (b) The field dependent mobilities of spin-coated, drop cast and E-field cast devices. (c) The photoluminescence spectra of drop-cast, E-field cast and spin coated devices excited at $510 \mathrm{~nm}$. 
interchain interaction. This suggests that there exists morphological features affecting mobility that are greater in size than the $10 \mathrm{~nm}$ limit of PL measurements and smaller in size than the $100 \mathrm{~nm}$ resolution of optical microscopy. Thus a complete understanding of charge transport requires information about these larger structures and their orientations with respect to the charge transport direction. We have preformed variety of surface and bulk morphological studies (detailed later) to understand these structures and how they affect charge carrier mobility in drop-cast, E-field-cast and spin cast samples.

\subsection{Thickness Dependence}

Since the mobilities were measured for thicknesses ranging from a few nanometers (spin-cast films $\sim 100 \mathrm{~nm}$ ) to few micrometers (drop-cast and E-field-cast $\sim 1-5 \mu \mathrm{m}$ ), it is worthwhile to see the effect of thickness on mobility. According to Scher and Montroll model the transit time scales with mobility in the dispersive region, ${ }^{28}$

$$
\mathrm{t}_{\mathrm{r}} \propto(\mathrm{E} / \mathrm{L})^{1 / \alpha} \rightarrow \mu \propto(\mathrm{E} / \mathrm{L})^{1 /(\alpha-1)}
$$

where $\mathrm{E}$ is the electric field, $\mathrm{L}$ is the device thickness and $\alpha$ $(0 \leq \alpha \leq 1)$ is the slope of the transient current in the log $\boldsymbol{i}$ - $\log \boldsymbol{t}$ plot. According to the S-M model, wit in the dispersive regime, the inverse of transit times for samples with different thicknesses plotted against E/L falls along a straight line with slope 2. In Fig. 9, 1/t tr for the drop-cast, E-field-cast and drop-cast devices are plotted against E/L. ${ }^{26}$ Even though the slopes of the lines of individual devices seem to have reasonable agreement with slope values of

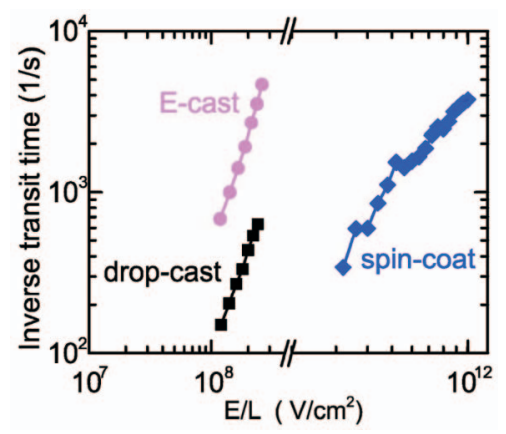

Fig. 9. Reciprocal transit times $\left(1 / t_{\text {tr }}\right)$ plotted against (E/L) at room temperature for the spin-coated, drop-cast and E-field cast. The slopes are in agreement with S-M theory however, they do not fall on the same line as required by the SM theory.
S-M model, they do not lie on the same line for the different processing conditions. If they lied on the same line, this would imply that the observed mobilities are solely due to the dispersive transport associated with the device thickness. The fact that they do not implies that there are morphological differences between the three films. Moreover, according to the S-M, mobility should increase with the increase in thickness. Our devices, contrast, exhibit the reverse of it. We thus attribute the differences in the mobility in these films are specific to the inherent morphology created by the processing conditions.

\subsection{Anisotropy in Mobility}

Fig. 10 summarizes the mobility perpendicular and parallel to the substrates. TrEL and TOF technique were used to measure the mobility perpendicular to the substrate for the spin and drop cast films respectively. Mobility parallel to the substrate was measured by the FET method using the bottom contact geometry for both spin cast and drop cast films. For the drop-cast films from chlorobenzene, the horizontal and vertical mobilities were of the same order of magnitude. For the spin cast films, the horizontal mobilities were orders of magnitude higher than of vertical mobilities measure by TrEL method. In other words, in the case of OLEDs (charge carrier transport is perpendicular to the substrate); the charge carrier mobilities were of the order of around $10-7 \mathrm{~cm}^{2} / \mathrm{Vs}$ while their counter part FET devices (charge transport is parallel to the substrate) have mobilities around $10^{-3} \mathrm{~cm}^{2} / \mathrm{Vs}$. The degree of anisotropy was solvent dependent. The horizontal mobilities in chlorobenzene being four orders of magnitudes higher than there ver-

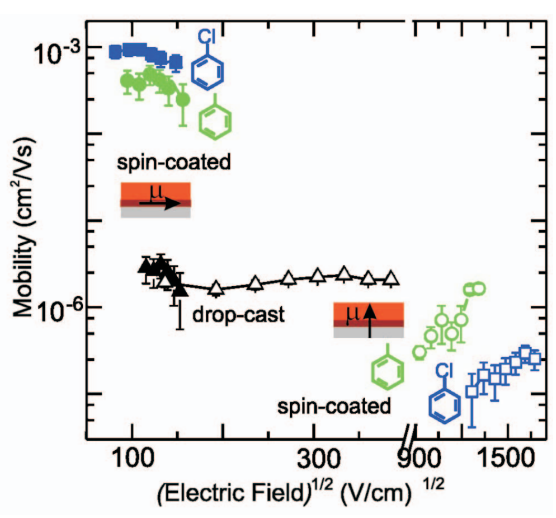

Fig. 10. Field dependent mobility of spin coated and drop-cast devices from toluene and chlorobenzene as measured by FET and OLED configurations 
tical mobilities while for spin-coated toluene devices, the difference was only 2 orders of magnitude. This anisotropy in the mobility has been attributed to the inherent morphology anisotropy in spin-cast films as opposed to earlier propositions of high charge carrier density, trapping or electric field effects. This argument was supported by our extensive bulk characterization by GI-WAXS, GI-SAXS, XRR, cross-section SEM measurements and surface studies by AFM and FESEM measurement.

\section{STRUCTURE}

Short range chain packing and mesoscale ordering were obtained by detailed investigation of WAXS and SAXS patterns respectively by using synchrotron and in-house facilities in the in-plane and out-plane geometry

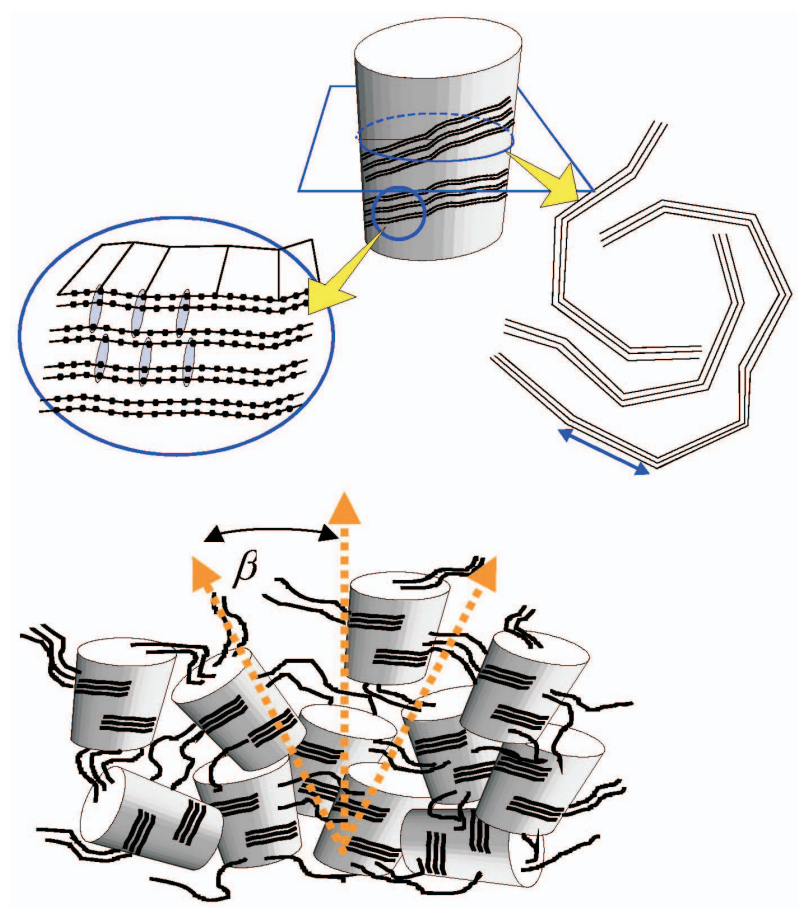

Fig. 11. Schematic structural model for drop-cast films. The cylindrical shape shown here is deduced from the FEM images. Top images show the horizontal cross section of MEH-PPV nano domains which exhibits fractal like coiling of MEH-PPV back bones greater than the persistent length (indicated by two way arrow). Top is the bilayer structure with uniaxial symmetry. Bottom image shows the preferentially oriented (towards surface normal) cylindrical domains composed of nanodomains. The remaining space is filled by the amorphous MEH-PPV chains. of drop-cast, E-field-cast and spin-cast devices. Based on these extensive investigations we propose a structural model for drop-cast ( $\mu \mathrm{m}$ thick films) and spin cast $(\mathrm{nm}$ thick films) in Fig. $11^{29}$ and Fig. 12.

GI-WAXS and GI-SAXS on drop-cast films exhibited anisotropic scattering profiles in the plane perpendicular to the substrate (out plane) and isotropic scattering profiles in the plane parallel to the substrate (in-plane). Drop-cast films (Fig. 11) seem to form locally stacked MEH-PPV chains of nano domains which extends up to mesoscale. From the top view, the isotropic WAXS and SAXS profiles were attributed to the MEH-PPV backbones (restricted by the persistent length ca.13 nm of MEH-PPV) which pass through the nanodomains of ordered packing with irregular fractal-like interfaces. From the side view, the bilayer peak of WAXS and the power law scattering of $r$ $=-4.2$ of SAXS was attributed to the ordered domain of smoother interfaces with bilayer structures. The spread angle $\beta$ is introduced to quantify the domain orientation, with disordered MEH-PPV chains filling the remaining space. The anisotropic orientation of ordered domains and its variation seems to be a function of processing conditions.

While all drop-cast films ordered domains preferentially orientated towards the surface normal, those formed from chlorobenzene seem to be smaller than those formed with toluene as the solvent. These smaller domains result in more order-disordered transition interfaces. We believe that domain interfaces hinder charge carrier mobility. Thus the increased domain interfaces in chlorobenzene films result in a direction hence lower mobility with respect to toluene based films. In the Bässler model, the increased number of domain interfaces is reflected in the higher position disorder parameter values for chlorobenzene relative to toluene drop-cast films. It is worth to note that the diffusion of charge carrier against the field directions is a dominant

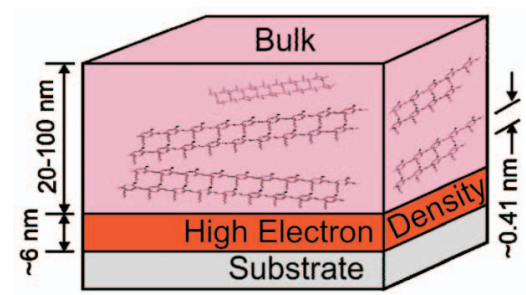

Fig. 12. Bulk structure of spin coated MEH-PPV films. This shows a $5 \mathrm{~nm}$ high electron density and missing aggregates (which are present in drop-cast films) and appearance of MEH-PPV backbones preferentially lying in the plane. 
effect in systems that have a high position disorder.

In the case of drop-cast thermally annealed films from toluene and chlorobenzene, these mesoscale domains seem to orient randomly through out the films and the anisotropy of preferential orientation toward surface normal present in the drop-cast films is lost. In addition, the bilayer packing structure (2.42 nm packing) in the drop-cast films is replaced with a bilayer structural packing of $1.75 \mathrm{~nm}$ spacing. Other than this, all other structural features observed are similar to the drop-cast films. The cross section SEM images of liquid nitrogen cracked drop-cast and drop-castannealed films showed particle (ca. 40-100 nm) like structures which indicated cluster of nanodomains that remain adhered during the cracking. This observation of fractographic features of drop-cast and drop-cast annealed films lead us to conclude that the main difference in the film morphology upon annealing is the randomization of nanodomains orientation. There is no significant difference between the structural features of drop-cast thermally annealed films from toluene and chlorobenzene.

A schematic diagram of bulk structure of spin-cast films is shown in Fig 12. The GI-WAXS reflection perpendicular to the surface at scattering vector $Q=15.2 \mathrm{~nm}^{-1}$ was attributed to the vertical inter-backbone packing with the spacing of $0.41 \mathrm{~nm}$, while the backbone chains remains parallel to the substrate. 27 This is consistent with the value obtained by the X-ray diffraction obtained previously. ${ }^{22}$ The weaker reflection parallel to the surface at scattering vector $\mathrm{Q}=10 \mathrm{~nm}^{-1}$ is attributed to the back bone repeat units of MEH-PPV. The average domain size of $1.4 \mathrm{~nm}$ is derived by Scherr formula from the diffuse peat at $\mathrm{Q} \sim$ $15 \mathrm{~nm}$ observed from the measurement of three angle integrated radial scans. This indicates that backbone order extends only up to three lattice constants. This confirms that the spin-cast polymer films are predominantly amorphous. The azimuth scans at $\mathrm{Q}=15.2 \mathrm{~nm}^{-1}$ suggested preferential backbone alignment parallel to the surface. Due to the smooth and unstructured GI-WAXS crystallinity inside the spin-cast devices was excluded.

The XRR profile of spin-cast films exhibited high frequency fringes modulated by the lower frequency oscillation. The high frequency Kiessig fringes reflected the film thickness and their extended obervability indicated the low surface roughness of our spin-cast films. The Pratt32 simulation software of XRR profiles suggested a $5 \mathrm{~nm}$ high electron film at the polymer substrates interface. The strength of this layer was found to vary with the solvents but not with the spin speed and film thickness.

The presence of $5 \mathrm{~nm}$ high electron density layer in spin-cast films might be due to the presence of higher degree of chain ordering at the interface. While this may not affect the vertical mobility measured in the TrEL measurements, this does have a strong influence in mobility measured by FET as most of the charge carriers are confined to the gate insulator polymer interface.

\section{CONCLUSIONS}

We have demonstrated that the charge carrier mobility varies by orders of magnitude depending on processing conditions and that there is a strong correlation between directional charge carrier mobility and morphology. MEHPPV polymer thick films $(\mu \mathrm{m})$ are found to have a mixture of amorphous and mesoscale crystalline domains consisting of nanodomains. The charge carrier mobility in these devices was found to increase with the decreasing number of domain interfaces. For example, (1) more homogenous structure in E-field-cast films exhibited higher mobility, (2) drop-cast films from toluene had higher mobility due to the presence larger preferentially oriented domains which provided less interfaces across the domains and (3) randomly oriented domains in drop cast annealed films exhibited more domain interfaces compared to that of drop-cast films and hence reduced the mobility. Spin cast films in the OLED structure exhibited the lowest mobility of the order of $10^{-6} \mathrm{~cm}^{2} / \mathrm{Vs}$. The highest mobility (of the order $10^{-3}$ $\mathrm{cm}^{2} / \mathrm{Vs}$ ) is obtained for spin cast films in an FET structure. This huge difference of mobility in the OLED and FET structures were attributed to the presence of a highly ordered layer at the gate insulator polymer interface.

\section{ACKNOWLEDGEMENTS}

We thank the National Synchrotron Radiation Research Centre (NSRRC) Hsinchu, Taiwan for valuable beam time. We thank our collaborators Dr. U. S. Jeng, Dr. C. H. Hsu, Dr. H. S. Hseu, and Dr. Lee of NSRRC, for their valuable contribution towards this work. We thank Prof. A. C. Su, National Sun Yat-Sen University, Kaohsiung, Taiwan for his SEM facilities and insightful discussions to arrive at the morphology parameters. We also thank our collaborator Dr. D. M. Smilgies, Cornell High Energy Synchrotron Source (CHESS), Cornell University, USA. We also thank our master students C. H. Tan, H. S. Chiu and C. C. Chang for their contribution to some of exciting work reviewed here. All the above collaborators have also contrib- 
uted as co-authors of the various publications with us.

Received May 17, 2010.

\section{REFERENCES}

1. Buroughes, J. H.; Bradley, D. D. C.; Brown, A. R.; Marks, R. N.; Mackey, K.; Friend, R. H.; Burn, R. L.; Holmes, A. B. Nature (London) 1990, 347, 539.

2. Malliaras, G.; Friend, R. Phys. Today 2005, 58, 53.

3. Forero, S.; Nguyen, P. H.; Brutting, W.; Schwoerer, M. Phys. Chem. 1999, 1, 1769.

4. Dai, L.; Winkler, B.; Dong, L.; Mau, A. W. H. Adv. Mater. 2001, 13, 915.

5. Shi, Y.; Liu, J.; Yang, Y. J. Appl. Phys. 2000, 87, 4254.

6. Liu, J.; Shi, Y.; Ma, L.; Yang, Y. J. Appl. Phys. 2000, 88, 605.

7. Nguyen, T.Q.; Kwong, R.C.; Thompson, M. E.; Schwartz, B. J. Appl. Phys. Lett. 2000, 76, 2454.

8. Nguyen, T. Q.; Martini, I. B.; Liu, J.; Schwartz, B. J. J. Phys. Chem. B 2000, 104, 237.

9. Jakubiak, R.; Collison, C. J.; Wan, W. C.; Rothberg, L. J. J. Phys. Chem. A 1999, 103, 2394.

10. Sirringhaus, H.; Brown, P. J.; Friend, R. H.; Nielsen, M. M.; Boohgaard, K.; Langegeld-Voss, B. M. W.; Splering, A. J. H.; Janssen, R. A. J.; Meijer, E. W.; Herwig, P.; de Leeuw, D. M. Nature (London) 1999, 410, 685.

11. Chen, S. H.; Su, A. C.; Chou, H. L.; Peng, K. Y.; Chen, S. A. Macromolecules 2004, 37, 167.

12. Saked, S.; Tal,; Roichman, Y.; Razin, A.; Xiao, S.; Eichen, Y.; Tessler, Adv. Mater. 2003, 15, 913.

13. Inigo, A. R.; Tan, C. H.; Fann, W. S.; Huang, Y. S.; Perng, G. Y.; Chen, S. A. Adv. Mater. 2001, 13, 504.

14. Tan, C. H.; Inigo, A. R.; Fann, W. S.; Wei, P. K.; Huang, Y. S.; Perng, G. Y.; Chen, S. A. Organ. Electron. 2002, 3, 85.
15. Spear, W. E. J. Non-Cryst. Solids 1969, 1, 197.

16. Bässler, H. Phys. Status Solidi B 1993, 175, 15.

17. Young, R. H.; Fitzgerald, J. J. J. Chem. Phys. 1995, 102, 6290. Extensive references listed in this paper on the charge transport in the dipolar medium by various groups including the work of P. M. Borsenberger and coworkers.

18. Pinner, D. J.; Friend, R. H. Tessler, N. J. Appl. Phys. 1999, $86,5116$.

19. Barth, S.; Muller, P.; Riel, H.; Seidler, P. F.; Reiz, W.; Vestweber, H.; Bässler, H. J. Appl. Phys. 2001, 89, 3711.

20. Muccini, M. Nature (Materials), 2006, 5, 605

21. Dimitrtakopolous, C. D.; Malenfant, P. R. L. Adv, Mater. 2002, 14, 99.

22. Yang, C. Y.; Hide, F.; Diaz-Garcia, M. A.; Heeger, A. J.; Cao, Y. Polymer 1998, 39, 2299.

23. Inigo, A. R.; Chiu, H.C.; Fann, W. S.; Huang, Y. S.; Jeng, U. S.; Lin, T. L.; Hsu, C. H.; Peng, K. Y.; Chen, S. A. Phys. Rev. B 2004, 69, 075201.

24. Inigo, A. R.; Chang, C. C.; Fann, W. S.; White, J. D.; Huang, Y. S.; Jeng, U. S.; Sheu, H. S.; Peng, K. Y.; Chen, S. A. $A d v$. Mater. 2005, 17, 1835.

25. Inigo, A. R.; Chiu, H. C.; Fann, W. S.; Huang, Y. S.; Peng, K. Y.; Chen, S. A. Synth. Met. 2003, 139, 581.

26. Huang, Y. F.; Inigo, A. R.; Chang, C. C.; White, J. D.; Huang, Y. S.; Jeng, U. S.; Hsu, C. H.; Su, A. C.; Peng, K. Y.; Chen, S. A. Adv. Funct. Mater. 2007, 17, 2902.

27. Huang, Y. F.; Chang, C. W.; Smilgies, D. M.; Jeng, U. S.; Inigo, A. R.; White, J. D.; Li, K. C.; Lim, T. S.; Li, T.; Chen, H. L.; Chen, S. A.; Chen, W. C.; Fann, W. S. Adv. Mater. 2009, $21,1$.

28. Scher, H.; Montroll, E. Phys. Rev. B 1975, 12, 2455.

29. Jeng, U. S.; Hsu, C. H.; Sheu, H. S.; Lee, H. Y.; Inigo, A. R.; Chiu, H. C.; Fann, W. S.; Chen, S. H.; Su, A. C.; Lin, T. L.; Peng, K. Y.; Chen, S. A. Macromolecules 2005, 38, 6566. 